\title{
Lorentz Symetry Breaking in Gravity and Dimensional Reduction
}

\author{
Marcelo Botta Cantcheff* \\ Instituto de Física Teórica (IFT/UNESP) \\ Rua Pamplona, 145 - Bela Vista \\ 01405-900 - São Paulo, SP - Brazil. \\ E-mail: bottalecbpf.br
}

We propose a modification of the standard Einstein theory in four dimensions, alternative to Ref. [2], since it is based on the first order (Einstein-Cartan) approach to General Relativity, whose gauge structure is explicit. A new topological term is introduced in the sector of coupling with matter and a Lorentz symmetry breaking is induced through a mechanism proposed in a recent paper [1]. An effective "planarity" may be observed in the resulting theory which resembles some aspects of holography. We finally remark some related proposals and open questions which are being developed and shall be discussed in forthcoming works.

Fifth International Conference on Mathematical Methods in Physics - IC2006

April 24-28 2006

Centro Brasileiro de Pesquisas Fisicas, Rio de Janeiro, Brazil

${ }^{*}$ Speaker. 


\section{Introduction.}

As mentioned in the abstract, this work consists partly, in the natural application to gravity of some ideas about an effective dimensional reduction in theories with a Chern Simons term in four dimensions, which clearly break Lorentz symmetry [1]. However, we further point out a novel suggestion about the possibility of quantizing four-dimensional gravity via a perturbative expansion around Chern-Simons theories in 2+1-dimensional hypersurfaces based on recent perspectives [3] which, in a certain sense, are related to the idea of holography.

A few years ago, a modification of Maxwell's electromagnetism in four dimensions has been proposed which considers a kind of Chern-Simons term in the action, $\int d x^{4} V_{\alpha} \varepsilon^{\alpha \beta \mu \nu} A_{\beta} F_{\mu v}$, where Lorentz symmetry is explicitly broken by an external vector, $V^{\mu}[6]$. There is a growing literature considering this proposal seriously, and exploring the possible origin and consequences $[8,9,10]$ of this approach.

In a recent work [1], we emphasized that broken Lorentz symmetry (abbreviated as BLS) could be obtained from physically realistic background configurations in non-linear relativistically invariant Electrodynamics. It was also pointed out that standard Chern-Simons terms (in $2+1$ dimensions [?]) are automatically present in a BLS action when we search for planar features (thus turning dimensional reduction unnecessary). In fact, the BLS action is actually a CS theory in $(2+$ 1)-dimensions embedded in $(3+1)$-dimensions, and by itself, it does not encode any information on the field-dependence in the direction of the external (for instance, space-like) vector $V:$ if $z$ is its affine parameter, i.e. $V=\frac{\partial}{\partial z}$, then we get a foliation of the spacetime in $(2+1)$-hypersurfaces $\Sigma_{z}$ parametrized by $z$ (and $V$ is orthogonal to each hypersurface ${ }^{1}$ ). Therefore, the BLS action may be written as

$$
S_{B L S}=\int_{0}^{L} d z S_{C S}\left[A(z), \Sigma_{z}\right]
$$

where

$$
S_{C S}\left[A(z), \Sigma_{z}\right]=\int_{\Sigma_{z}} \mathscr{L}_{C S}=\int_{\Sigma_{z}} A(z) \wedge d A(z)
$$

is the Chern-Simons action for the 1-form gauge field $A(z)$ on a three-dimensional manifold $\Sigma_{z}$. Thus, the dependence of this field on the parameter $z$ is not determined by this theory. It only has to satisfy usual convergence conditions. For example, if the interval $(0, L)$ extends to $(-\infty,+\infty), A(z)$ has to be an square-integrable function $\left(A \in L^{2}(\Re)\right)$. In this sense, we can interpret the BLS action simply as a sum of Chern-Simons theories on manifolds $\Sigma_{z}$. Remarkably notice that this describes an eventual situation of confinement of the electromagnetic field (photon) into a $(2+1)$-manifold, which does not result from a constraint of the charged matter into a planar sample. The present work actually constitutes an attempt of naturally extending to gravity some of these ideas.

There is an additional (actually, the main one) theoretical motivation for our construction. Recently, a Chern Simons modification of gravity in four dimensions via a BLS term was introduced by Jackiw and Pi [2] in a similar way as that for Electrodynamics. However, this approach is based on the second order formulation of general relativity, where the most relevant aspects of the Maxwell theory, related to the gauge structure, are hidden. In this work, we alternatively formulate

\footnotetext{
${ }^{1}$ Notice that if the space-time (or the space-time region considered in the integration) is simply connected, the condition of existence of this $z$-coordinate is equivalent to gauge invariance of the action, namely $d V=0$.
} 
a BLS/CS deformation of standard gravity but now based on first order Cartan's formalism (see Appendix), which treats the Riemann Tensor as an standard gauge curvature for the spin connection which may be viewed as a gauge variable of $S O(1,3)$. Thus, such an approach is closer in spirit to the Chern-Simons deformation of Electrodynamics [6].

The model we are going to build up here assumes a non-linear (but relativistic) Geometrodynamics which induces a modification of this kind (BLS) on the standard Einstein theory [2][7]. Furthermore, it is argued that BLS does not need to be introduced by hand, but it can naturally appear in some realistic physical situations, according to the philosophy adopted for Electrodynamics [1](and consequently, a CS term will be induced on a family of embedded hypersurfaces). For example, in the presence of background gravitational fields and/or when non-uniform distributions of matter are considered .

Finally, we point out here an interesting novel possibility (which is, perhaps, our main observation), associated with the behavior of the theory in a sort of strong coupling limit, which resembles holography, since the free Fock space could be built from three dimensional theories of gravity, and the full Geometrodynamics would be recovered perturbatively about these states.

The idea of treating general relativistic theories with a perturbative expansion around a topological theory has been recently put forward in the quantum gravity literature [3] (and its viability was later investigated in the context of conventional Yang-Mills theory on flat spacetime [4]). This idea is based on the fact that GR can be written as a modified topological BF theory. So, the proposal of [3] is to construct quantum gravity through a perturbative expansion around the exact topological BF theory. So we wish to give a step further in this direction by suggesting that this topological theory could be an ordinary $2+1$-dimensional Chern-Simons theory. Thus, in principle, by considering CS modified General Relativity and a proper BLS background, we could recover the bulk quantum gravity perturbatively. In such a sense, we could recognize a sort of "holographic" structure in this perturbation scheme.

Below, we shall briefly comment the lines along which such ideas could be implemented in practice.

\section{Chern-Simons modified gravity and discussion}

We use both the abstract index notation ${ }^{2}$ (see Appendix for more details), and forms notation (by omiting abstract sub-indices) whenever it is convenient. So, greek indices $\mu, v, \ldots{ }^{3}$ denote the element of a tetrad (vierbein) basis $\left(e_{a}\right)^{\mu}$, and consequently components of any tensor in this basis.

Let us propose a Chern Simons modification of General Relativity (GR) in the first order formalism (see Apendix):

$$
S[e, w, \phi]=\frac{1}{2 \kappa^{2}} \int_{M} d x^{4}\left(e^{\mu} \wedge e^{v} \wedge^{*} R_{\mu v}+\tau R \wedge R\right)+S_{\text {matter }}[\phi]
$$

where the two form $R=d w^{\mu}{ }_{v}+w^{\mu}{ }_{\alpha} \wedge w^{\alpha}{ }_{v}$ is defined as the $S O(1,3)$-field strength for the gauge field $w_{a v}^{\mu}$. The scalar $\tau$ is, in principle, a pointwise function of the geometry observables, as the

\footnotetext{
${ }^{2}$ Abstract index notation is a mathematical notation for tensors and spinors, which uses indices to indicate their type. Thus the index isn't related to any basis or coordinate sytem.

${ }^{3}$ Which are rised and lowered with the Minkowski metric $\eta_{\mu v}$.
} 
curvature tensor, and of some "extra" (matter) field, denoted by $\phi$. So, the embedding variable is considered itself as a dynamical variable rather than a fixed external quantity.

Notice then that Lorentz symmetry is preserved in a fundamental sense. If one assumes that a more fundamental unified theory of matter and gravity is non-linear, a saddle point expansion about background solutions typically shall give origin to a BLS term (and even spontaneous BLS terms) with a fixed $\tau$ of this form. This may be easily argued for sufficiently generic non linear (toy) theories, in similar ways than that for Electrodynamics (see Ref. [1]).

The first term corresponds to the usual Einstein-Hilbert action in the first order representation (Einstein-Cartan), the second one is the Chern-Simons modification, where we have assumed that the coefficient $\tau$ may depend on the curvature components and/or other (matter) fields. In such a sense, this term should be viewed as an interaction term. This may be expressed as

$$
S_{B L S}=-2 \int_{M} d x^{4}\left(d \tau \wedge \mathscr{L}_{C S}\right)
$$

where

$$
K^{a} \equiv\left({ }^{*} \mathscr{L}_{C S}\right)^{a} \equiv \varepsilon^{a b c d} w_{b v}^{\mu} R_{c d \mu}^{v}
$$

is the Chern Simons current density whose divergence is the topological number called the gravitational Pontryagin density, $P \equiv \frac{1}{2}{ }^{*} R R \equiv \frac{1}{2}\left(\varepsilon^{a b c d} R_{a b \mu}^{v} R_{c d v}^{\mu}\right)$. Notice remarkably that the matter fields are coupled to the geometry through the topological term.

For simplicity, let us restrict ourselves to the case when $\tau$ does not depend on the geometric variables $e_{\mu}^{a}, w_{a v}^{\mu}$; so, varying this action with respect to $e_{a}^{\mu}$, we have:

$$
e_{\mu}^{a} R_{a b}^{\mu v}=\kappa^{2}{T^{\prime}}_{b}^{v}=\kappa^{2} e^{v a} T^{\prime}{ }_{a b}
$$

where one has defined $T^{\prime}{ }_{a b}:=T_{a b}+g_{a b}\left(T_{c d} g^{c d}\right) / 2, T_{a b}$ being the energy momentum tensor, and the constant $\kappa$ is related to the gravitation constant, $G$, by $\kappa^{2}=8 \pi G$. Varying now this action with respect to $w_{a v}^{\mu}$

$$
d \wedge e^{\mu}+w^{\mu}{ }_{v} \wedge e^{v}=\Theta^{\mu}
$$

which are the usual Einstein Cartan equations for the geometry but with an effective torsion which depends on the external field in the form:

$$
\Theta^{\mu}=2 \kappa^{2 *}\left(d \tau \wedge R^{\mu \nu} \wedge e_{v}\right)
$$

If we solve first for the spin coefficients $w_{a v}^{\mu}$ in terms of $e_{a}^{v}$ and $\nabla_{a} \tau$ in Eq. (2.5) and replace into (2.4), we recover the modified Einstein equation for the tetrad $e_{a}^{v}$ (or, equivalently, for the metric $g_{a b}$ ) obtained in the Jackiw-Pi approach [2].

We may already point out the main behavior we are interested:

The gradient of the external field $\tau$ dictates the coupling of the geometric degrees of freedom with the Chern-Simons 3-form Lagrangian $\mathscr{L}_{C S}=R^{\mu v} \wedge w_{\mu v} \equiv R \wedge w$. In fact, this may be expressed as $\nabla_{a} \tau \equiv g^{2} V_{a}\left(\Rightarrow g^{2}=|d \tau|\right)$ where $V$ is a unit vector in the gradient direction. In the limit $g \rightarrow 0$ the standard torsion-free Einstein theory is recovered and, on the other hand, when 
$g \rightarrow \infty$, the CS term governs the dynamics of the geometry! In fact, notice that if $g$ is considered nearly constant and we rescale the spin connection and define the new gauge variable $A^{\mu \nu} \equiv g w^{\mu v}$ and the field strength $F^{\mu v} \equiv d A^{\mu v}+g^{-1} A^{\mu \alpha} \wedge A^{\beta v} \eta_{\alpha \beta}$, the action (2.1) may be written as:

$$
S_{G r a v}[e, A, \phi]=\int_{M} d x^{4}\left(g^{-1} e^{\mu} \wedge e^{v} \wedge^{*} F_{\mu v}-\frac{1}{2} V \wedge A \wedge F\right),
$$

where we have used the equivalence of the second term of (2.1) with the Chern-Simons form. Thus, we can see in this expression that, in this case, the first term is a first order perturbation in $g^{-1}$ while the second one, a Chern-Simons action, is considered the free kinetic term (zeroth order).

In this case, by a similar argument as that for Electrodynamics (shown in the Introduction), the theory becomes a $2+1$-dimensional topological theory, which is precisely equivalent to $3 d$ gravity, is exactly soluble and its quantization is well understood [5] ${ }^{4}$. In fact, on each level(hyper)surface of the field $\tau(\phi(x))$ ), we have a Chern Simons action for the connection $w^{\mu v}$ in the group $S O(1,3)$, which contains the Lorentz-Poincaré group $I S O(1,2)$ if the dreibein $E^{\hat{\mu}}(\hat{\mu}, \hat{v}=$ $0,1,2)$, the gauge field associated with translations on those hypersurfaces, is identified with $w^{\hat{\mu}, 3}$ and the spin connection $w^{\hat{\mu} \hat{v}}$ is the gauge field associated whith $S O(1,2)$.

Let us remark here an important open question which has to be investigated: could this strong/weak behavior be interpreted as duality? If the answer is "yes" we might claim an holographic structure in the most obvious and direct sense.

The third term of (2.1) encodes the dynamics of the field $\phi$ but we do not give here any explicit Lagrangian. However we can notice that in general, the gravitational Pontryagin density constitutes a source for the equation of motion of $\phi$, i.e:

$$
\frac{1}{\tau^{\prime}(\phi)} \frac{\delta S_{\text {matter }}[\phi]}{\delta \phi}=N
$$

where we have assumed that $\tau$ is only a pointwise funtion of $\phi$.

In particular, if we consider the simplest case, where $\tau \equiv \phi$, and $S_{\text {matter }}[\phi]$ is a Klein-Gordon field on a curved spacetime:

$$
\left(\nabla^{a} \nabla_{a}-m^{2}\right) \phi=N
$$

Since we should be particularly interested in applying these ideas to the context of solutions such as black holes, let us discuss some aspects of static spherically symmetric solutions of this problem. It is clear that, by virtue of the Hamilton equations, static solutions correspond to solutions which are local extrema of the Hamiltonian density and generically, local minima ${ }^{5}$. So, if we consider spherical symmetry and assimptotic boundary conditions of flatness of the spacetime, and vanishing of the field $\phi$ (when $r \rightarrow \infty$ ), then the solution of (2.9) is unique and the topological nature of the charge $N$ guarantees that this local minimum is stable.

Let for instance focus on solutions of (2.9) with $N=0$. To solve this equation, at least assimptotically, we make the following ansatz: the operator $\nabla^{a} \nabla_{a}$ coincides with the Klein Gordon operator on a Schwarzchild spacetime. Then, we expand $\phi$ in spherical harmonics and write this

\footnotetext{
${ }^{4}$ In such a theory of gravity there are no local degrees of freedom and all solutions are conformally flat.

${ }^{5} \mathrm{~A}$ convenient choice of a potential term may be added to $S_{\text {matter }}[\phi]$ in order to ensure this.
} 
wave equation for each mode in the form $r^{-1} f(r, t) Y_{l m}(\theta, \beta)$; but the solution we are interested (with $l=0$ and independent of $t$ ) satisfies:

$$
-\frac{\partial^{2} \phi}{\partial r_{*}^{2}}+(1-2 M / r)\left(2 M / r^{3}+m^{2}\right) \phi=0
$$

where the coordinate $r_{*} \equiv r+2 M \ln (r / 2 M-1)$ has been defined. The second term of this equation may be seen as an effective potential. $M$ is the mass of the black hole which is independent of $m$ since the energy-momentum tensor in Eq. (2.4), may have other contribution apart from $\phi$ (which contributes to the torsion), and it shall be supposed to be large enough (i.e $M>>m$ ) to precisely ensure consistency with equation (2.10). So, straightforwardly we get that, for $r_{*} \rightarrow \infty$ (i.e $r \rightarrow \infty$ ), the radial derivative is negligible which means, by virtue of equation (2.5), that the BLS contribution is also negligible. Consequently, we have standard Einstein-Cartan equations, which finally yield the Schwarzchild solution, which proves the consistency with our inicial ansatz. This is an example of how a spherically symmetric soliton-like solution may be found in this context, that can be taken as a suitable vacuum for building up a probably more convenient quantization scheme.

Recall that there is no reason for taking this type of linear action for $\phi$ and other theories with interesting properties could be considered in order to obtain the desired behavior. From the point of view of this "holographic" quantization discussed above, the most interesting possibilities that we want to consider are those actions for $\phi$ such that the radial derivative decreases with $r$ with appropriate rapidity in order to have a well defined perturbative framework (i.e in order to have properties such as adiabaticity). Notice that in this case (with spherical symmmetry) the twodimensional surfaces where the Chern-Simons theories live are spheres $S_{r}^{2}$. So, as $r$ is smaller, the CS theory is more important in the general dynamics. In a forthcoming work, we are going to analyze these theories and solutions and explore this possibility of quantization in more detail.

Finally, another open question to be analyzed elsewhere is if the $N$-vacua are related, at least assimptotically (via the Chern-Simons modification), to deformations of the Schwarzchild solution, such as Kerr or Reissner-Nordstrom metrics.

\section{Apendix: The Abstract index Notation and Einstein-Cartan formalism}

In this work, we shall use the abstract index notation [11]; namely, a tensor of type $(n, m)$ shall be denoted by $T_{b_{1} \ldots . . b_{m}}^{a_{1} \ldots a_{n}}$, where the latin index stand for the numbers and types of variables on which the tensor acts and not as the components themselves on a certain basis. Then, this is an object having a basis-independent meaning. In contrast, greek letters label the components, for example $T_{\alpha}^{\mu v}$ denotes a basis component of the tensor $T_{c}^{a b}$. We start off with the Cartan's formalism of GR. We introduce [11] an orthonormal basis of smooth vector fields $\left(e_{\mu}\right)^{a}$, satisfying

$$
\left(e_{\mu}\right)^{a}\left(e_{v}\right)_{a}=\eta_{\mu v}
$$

where $\eta_{\mu \nu}=\operatorname{diag}(-1,1,1,1)$. In general, $\left(e_{\mu}\right)^{a}$ is referred to as vielbein. The metric tensor is expressed as

$$
g_{a b}=\left(e^{\mu}\right)_{a}\left(e^{v}\right)_{b} \eta_{\mu v}
$$


$>$ From now on, component indices $\mu, v, .$. will be raised and lowered using the flat metric $\eta_{\mu v}$ and the the abstract ones, $a, b, c \ldots$ with space-time metric $g_{a b}$.

Now we define the Ricci rotation coefficients, or spin-connection,

$$
\left(w_{\mu v}\right)_{a}=\left(e_{\mu}\right)^{b} \nabla_{a}\left(e_{v}\right)_{b}
$$

where $w_{a \mu v}$ is antisymmetric what, together with (3.1), is equivalent to the compatibility condition

$$
\nabla_{a} g_{b c}=0
$$

From (3.3), we have

$$
\nabla_{a} e_{b}^{\mu}=-w_{a}^{\mu v} e_{v b}
$$

whose antisymmetric part is (by adopting the convention of anti- symmetrization: $(\ldots)_{[a b]}=\left((\ldots)_{a b}-\right.$ $\left.\left.(\ldots)_{b a}\right) / 2\right)$,

$$
\nabla_{[a} e_{b]}^{\mu}=-w_{[a}^{\mu v} e_{b]}^{\alpha} \eta_{v \alpha} .
$$

In the original Einstein formulation of GR, the connection is assumed to be torsion-free. This is expressed by:

$$
\partial_{[a} e_{b]}^{\mu}=-w_{[a}^{\mu v} e_{b]}^{\alpha} \eta_{v \alpha} .
$$

The components of the Riemman's tensor in this orthonormal basis are given as follows

$$
R_{a b}^{\mu v}:=2 \partial_{[a} w_{b]}^{\mu v}+2 w_{[a}^{\mu \rho} w_{b]}^{\sigma v} \eta_{\rho \sigma} .
$$

Equations (3.7) and (3.8) are the structure equations of GR in Cartan's framework.

Einstein's equation in this framework reads

$$
e_{\mu}^{a} R_{a b}^{\mu v}=\kappa^{2} e^{v a} T^{\prime}{ }_{a b},
$$

where one has defined $T^{\prime}{ }_{a b}:=T_{a b}+g_{a b}\left(T_{c d} g^{c d}\right) / 2, T_{a b}$ being the energy momentum tensor, and the constant $\kappa$ is related to the gravitation constant, $G$, by $\kappa^{2}=8 \pi G$.

Equations (3.5) and (3.9) are a system of coupled first-order non-linear equations for the variables $(e, w)$ which determine ${ }^{6}$ the dynamics of GR. Metric and covariant derivative result finally defined in terms of these variables as seen from (3.2) and (3.5).

This yields the so-called "Einstein-Cartan formalism"; we obtain, thereby, a first order EinsteinHilbert action which can be expressed as

$$
S=\frac{1}{2 \kappa^{2}} \int d x^{D} e R_{a b}^{\mu v} e_{\mu}{ }^{a} e_{v}{ }^{b},
$$

where $e=(-\operatorname{det} g)^{1 / 2}=\operatorname{det}\left(e_{a}^{\mu}\right)$. If we wish to consider a non-vanishing cosmological constant, $\Lambda, R_{a b}^{\mu v}$ must be replaced by

$$
R_{a b}^{\mu v}+\Lambda e_{a}^{[\mu} e_{b}^{v]}
$$

\footnotetext{
${ }^{6}$ Together with the antisymmetry condition for $w_{a}$.
} 


\section{Acknowledgements}

The author thanks A.L.M.A. Nogueira and José A. Helayël Neto for fruitful discussions. CNPq is especially acknowledged for the invaluable financial help.

\section{References}

[1] M. Botta Cantcheff, Lorentz symmetry breaking and planar effects from non-linear electrodynamics, Eur. Phys. Jour. C 46 (3127) 2006 [hep-th/ 0411254 ].

[2] R. Jackiw, S.-Y. Pi, Chern-Simons modification of general relativity, Phys. Rev. D 68 (104012) 2003, [gr-qc/0308071]

[3] L. Freidel, A. Starodubtsev, Quantum gravity in terms of topological observables, [hep-th/0501191].

[4] C. Rovelli, S. Speziale, On the perturbative expansion of a quantum field theory around a topological sector, [gr-qc/0508106].

[5] E. Witten, (2+1)-Dimensional gravity as an exactly soluble system, Nucl. Phys. B 311 (46) 1988.

[6] S. M. Carroll, G. B. Field, R. Jackiw, Limits on a Lorentz and parity violating modification of electrodynamics, Phys. Rev. D 41 (1231) 1990.

[7] V. A. Kostelecky, Gravity, Lorentz violation, and the standard model, Phys.Rev. D 69 (105009) 2004, [hep-th/0312310].

V. A. Kostelecky, Lorentz violation and gravity in proceedings of 3rd Meeting on CPT and Lorentz Symmetry (CPT 04), Bloomington, Indiana, 4-7 Aug 2004,

http: //www.indiana.edu/ cpt04/, [hep-ph/0412406].

V. B. Bezerra, C.N. Ferreira, J. A. Helayel-Neto, Lorentz-breaking effects in scalar-tensor theories of gravity, Phys.Rev. D 71 (044018) 2005, [hep-th/ 0405181 ].

[8] F.R. Klinkhamer, A CPT anomaly, Nucl. Phys. B 578 (277) 2000, [hep-th/9912169].

[9] C. Adam, F.R. Klinkhamer, Causality and CPT violation from an abelian Chern-Simons like term, Nucl.Phys. B 607 (247) 2001, [hep-ph/0101087].

C. Adam, F.R. Klinkhamer, Causality and radiatively induced CPT violation, Phys. Lett. B 513 (245) 2001 , [hep-th/0105037].

A. Kostelecky, R. Lehnert, Stability, causality, and Lorentz and CPT violation, Phys.Rev. D 63 (065008) 2001, [hep-th/0012060].

A. A. Andrianov, R. Soldati, L. Sorbo, Dynamical Lorentz symmetry breaking from $(3+1)$

Axion-Wess-Zumino model, Phys. Rev. D 59 (025002) 1999 [hep-th/9806220].

R. Jackiw, A. Kostelecky, Radiatively induced Lorentz and CPT violation in electrodynamics, Phys. Rev. Lett. 82 (3572) 1999, [hep-ph/9901358].

J. -M. Chung, B. K. Chung, Induced Lorentz and CPT violating Chern-Simons term in QED: Fock-Schwinger proper time method., Phys.Rev. D 63 (105015) 2001, [hep-th / 0101097 ].

J. -M. Chung, Lorentz and CPT violating Chern-Simons term in the formulation of functional integral., Phys.Rev. D 60 (127901) 1999, [hep-th/9904037].

M. Perez-Victoria, Exact calculation of the radiatively induced Lorentz and CPT violation in QED, Phys. Rev. Lett. 83 (2518) 1999, [hep-th/9905061]. 
G. Bonneau, Regularization: many recipes, but a unique principle, Ward identities and normalization conditions: the case of CPT violation in QED, Nucl. Phys. B 593 (398) 2001 , [hep-th/ 0008210 ]. M. Perez-Victoria, Physical (ir)relevance of ambiguities to Lorentz and CPT violation in QED, JHEP 0104 (032) 2001, [hep-th/0102021].

[10] H. Belich, M.M. Ferreira Jr., J.A. Helayël-Neto, M.T.D. Orlando, Classical solutions in a Lorentz violating Maxwell-Chern-Simons electrodynamics, Phys. Rev. D 67 (125011) 2003 [hep-th/0212330].

H. Belich, M.M. Ferreira Jr., J.A. Helayël-Neto, M.T.D. Orlando Dimensional reduction of a Lorentz and CPT violating Chern-Simons model, Phys.Rev. D 68 (025005) 2003, [hep-th/ 0301224 ].

H. Belich, M.M. Ferreira Jr., J.A. Helayel-Neto Dimensional reduction of an abelian Higgs-Lorentz violating model, Nucl.Phys.Proc.Suppl. 127 (105) 2004 [hep-th/ 030508 9].

[11] R. M. Wald General relativity, University of Chicago Press, Chicago and London, 1984. 